P179 (continued)

Objective: To examine the relationship between baseline and post accelerometer derived physical activity (PA) in 910 year old children participating in iCook $4-\mathrm{H}$, a 16 -week, 5-state, randomized control family centered childhood obesity prevention program.

Design, Setting and Participants: This family focus intervention was designed to promote PA through lifestyle modifications. Between group differences in minutes-per-day and intensity of PA were determined on 76 (Intervention $n=51$; Control $n=25$ ) accelerometer compliant participants.

Outcome Measures and Analysis: All subjects wore an accelerometer (Actigraph GT3X+) for 7 days at baseline and post intervention assessment. Mean daily minutes of accelerometer derived sedentary time (ST) and PA including light (LPA), moderate (MPA), vigorous (VPA) and moderate-to-vigorous (MVPA) were evaluated during waking hours. A multivariate test, Wilks' Lambda, was used to determine the between group differences of PA from pre to post. Statistical significance was set at $\mathrm{p} \leq 0.05$. Results: There were no significant differences between groups from baseline to post intervention in any intensity category of PA (mean min/day \pm SD; p-value) ST baseline treatment [BT] $546 \pm 57$, baseline control ([BC] 547 \pm 62 , post treatment [PT] $580 \pm 63$, post control [PC] $563 \pm 66$; $\mathrm{p}=0.28)$, LPA (BT $244 \pm 48$, BC $239 \pm 50$, PT $217 \pm 52$, PC $228 \pm 50 ; \mathrm{p}=0.25$ ), MPA (BT $33 \pm 10$, BC $36 \pm 12$, PT $28 \pm 9$, PC $33 \pm 13 ; \mathrm{p}=0.61$ ), VPA (BT $16 \pm 9$, BC $18 \pm 9$, PT $14 \pm 7$, PC $16 \pm 8 ; \mathrm{p}=0.74$ ), and MVPA (BT $50 \pm 17$, BC $54 \pm 19$, PT $42 \pm 14$, PC $49 \pm 20 ; \mathrm{p}=0.58)$.

Conclusions and Implications: Although there was not a significant increase in any category of physical activity due to the lifestyle intervention, iCook 4 -H was able to encourage participants to maintain current activity levels. Funding: USDA.

\section{P180 Challenges in Measuring School Lunch Consumption Using Digital Photography in Urban Elementary School Students}

Randi Wolf, PhD, wolf@tc.columbia.edu, Teachers College, Columbia University, 525 West 120th Street, Box 137, New York, NY 10027; T. Ammeter, BS; P. Koch, EdD, RD; C. Uno, MLIS; I. Ang, MA; H. L. Gray, PhD, RD; I. Contento, PhD

Objective: To describe challenges and potential solutions to using digital photography for evaluation of school lunch consumption.

Theory, Prior Research, Rationale: Accurately measuring food intake in school cafeterias poses a variety of challenges. Digital photography is increasingly used as an objective measurement of food selection, intake, and leftovers, and has been shown to be valid, reliable, and cost-effective.

Conclusions and Implications: A variety of challenges can be anticipated when digital photography is used to measure meal consumption in real-world settings and thorough study protocols can ensure accurate and reliable data.
Target Audience: As part of an evaluation of a large integrated seed-to-table gardening program, we collected digital photos of school lunch on $\sim 300$ K-8 students from one low-income NYC school, 3 days each in spring 2013 and spring 2014.

Description: Digital photographs were taken of individual trays before and after lunch. Any food from home was also included in the photographs. Side-by-side pairs of photos (before- and after-lunch) were visually assessed to determine food selection and consumption.

Evaluation: Challenges included: capturing food shared, estimating amounts consumed of food in opaque packaging (e.g., apple slices, pretzels) or beverages (e.g., milk from school, drink containers from home), assessing food that was consumed while still on lunch line (before-lunch photograph) or food thrown away before the after-lunch photograph, ensuring trays were properly labeled with unique IDs, ensuring high quality photographs (taken without flash) taken at consistent angles, and ensuring brand names of packaged foods were oriented towards camera. Protocols that addressed each challenge were developed for future studies.

Funding: Edible Schoolyard New York City.

\section{P181 How Do Consumers Use Nutrition Labels on Food Products?}

Yuanting Zhand,PhD, Yuanting.Zhang@fda.hhs.gov, FDA, 5100 Paint Branch Pkwy, 2C-102, College Park, MD 20740

Objective: To understand how consumers use food labels and the trends of changes in consumer food label usage styles. To our knowledge, the present study was the first that examines food label usage styles with four compatible nationally representative surveys.

Design, Setting and Participants: We compared four cross-sectional Health \& Diet Surveys (HDS) - HDS 1994 $(\mathrm{n}=1,945), 1995(\mathrm{n}=1,001), 2002(2,743)$ and 2008 $(\mathrm{n}=2,584)$. United States Adults (18+).

Outcome Measures and Analysis: Eight food label usage questions and an index summing up these eight questions. We used cluster analysis, multinomial logistic, ordinary least squares regression modeling and nonparametric statistics.

Results: As speculated, the eight label questions were grouped into two categories, which were generally in line with our label usage for shopping decisions or dietary decisions (e.g., meal planning) classifications. Regarding label usage styles, consumers paid either equal or more attention to label information for dietary decisions rather than for shopping decisions in 2008 as compared to previous years 1995 and 2002. More people used labels for all purposes around the implementation of NLEA in 1994 and 1995 as compared to $2008(\mathrm{P}<.001)$. Females, highly educated (some college or above), young old (aged 5059), consumers with any health issue, and frequent label users were significantly more likely to use food labels for all kinds of purposes. 